accepted for publication in Physical Review E 62, (2000)

\title{
Crossover phenomenon in self-organized critical sandpile models
}

\author{
S. Lübeck* \\ Theoretische Tieftemperaturphysik, Gerhard-Mercator-Universität Duisburg, \\ Lotharstr. 1, 47048 Duisburg, Germany
}

(Received 10. April 2000)

\begin{abstract}
We consider a stochastic sandpile where the sand-grains of unstable sites are randomly distributed to the nearest neighbors. Increasing the value of the threshold condition the stochastic character of the distribution is lost and a crossover to the scaling behavior of a different sandpile model takes place where the sand-grains are equally transferred to the nearest neighbors. The crossover behavior is numerically analyzed in detail, especially we consider the exponents which determine the scaling behavior.
\end{abstract}

64.60.Ht,05.65.+b,05.40.-a

\section{INTRODUCTION}

Crossovers between different universality classes are well known from equilibrium phase transitions. Similar crossover phenomena are also known from nonequilibrium systems which exhibit self-organized criticality [1]. For instance the forest-fire model of Drossel and Schwabl [2] displays a crossover to a percolation like scaling behavior if one introduces an immunity parameter which prevents trees from burning [3]. Another example is the directed Abelian sandpile model of Dhar and Ramaswamy [4]. Introducing a second stochastic toppling condition the system changes to a directed percolation like scaling behavior [5].

In this paper we investigate a crossover between the stochastic sandpile model introduced by Manna [6] and the Zhang sandpile model [7]. Following Ben-Hur and Biham [8] the crossover connects the different universality classes of undirected sandpile models (the energy or sand-grain transfer of the nearest neighbors is isotropic, e.g. the Zhang model) and undirected on average sandpile models (the energy transfer is isotropic on average only, e.g. the Manna model). Considering the Manna model the crossover takes place just by increasing the threshold value which determines the dynamics, i.e., no additional parameter has to be introduced.

In the next section we briefly remind the distinctive characteristics of the Zhang model. These characteristics will allow us in the following to identify the typical Zhang scaling behavior. Then we describe in section III the Manna model for different values of the threshold condition. The crossover behavior between both models is investigated in section IV. A summary closes the paper.

\section{THE ZHANG MODEL}

Consider the Zhang model [7] on a two dimensional square lattice of linear size $L$. A continuous value $E_{\underline{r}} \geq 0$ representing the energy is associated to each lattice site $\underline{r}$. A configuration $\left\{E_{\underline{r}}\right\}$ is stable if $E_{\underline{r}}<E_{\mathrm{c}}$ for all lattice sites $\underline{r}$. For the sake of simplicity we choose in all simulations $E_{\mathrm{c}}=1$. A quantum of energy $\delta$ is added to a randomly chosen lattice site $\underline{r}$, i.e.,

$$
E_{\underline{r}} \rightarrow E_{\underline{\underline{r}}}+\delta .
$$

We consider in this work especially the slow driving limit $\delta \ll E_{\mathrm{c}}$. For $\delta \rightarrow 0$ all lattice sites grow parallel and the Zhang model corresponds to the conservative limit of the "spring block" model of Christensen and Olami [9].

In the case that due to the perturbation a site exceeds the critical value $E_{\mathrm{c}}$ an activation event will occur, the unstable site relaxes to zero, and the energy is added to the nearest neighbors, i.e.,

$$
\begin{gathered}
E_{\underline{r}} \rightarrow 0, \\
E_{\underline{r}, N N} \rightarrow E_{\underline{r}, N N}+\frac{E_{\underline{r}}}{4} .
\end{gathered}
$$

The transferred energy may activate the neighboring sites and thus an avalanche of relaxation events may take place. Energy may leave the system only at the boundary. Since the energy of unstable sites is equally transfered to the nearest neighbors it was argued that the Zhang model belongs to the universality class of undirected sandpile models [8]. It was expected that the Zhang model and the well-known Bak-Tang-Wiesenfeld (BTW) model [1] belong to the same universality class. But the scaling behavior of the BTW avalanches is complex and is not understood. Although most authors agree upon a breakdown of simple scaling the interpretation of the numerically obtained data is still controversial among the different groups (see for instance [10 13]). However, we use in the following the classification ansatz of [8] and denote the universality class of the Zhang model as the class of undirected sandpile models.

The avalanches are characterized by several physical properties like the size $s$ (number of relaxation events), the area $a$ (number of distinct toppled sites), the time $t$ (number of parallel updates until the configuration is stable), the radius $r$ (radius of gyration), etc. In the critical steady state the corresponding probability distributions should obey power-law behavior [1]

$$
P_{x}(x) \sim x^{-\tau_{x}}
$$

characterized by the avalanche exponents $\tau_{x}$ with $x \in$ $\{s, a, t, r\}$.

The Zhang model was intensively investigated in the last years (see for instance [7, 14 18]). A characteristic 
property of the Zhang model is the concentration of the steady state energy distribution $p(E)$ around $z$ distinct peaks, where $z$ is the number of nearest neighbors of the lattice [7, 14, 15, 17]. The peaks are located at multiples of $(z+1) / z^{2}$ and the height of the peaks diverges in the thermodynamic limit $L \rightarrow \infty$ (see [17] and references therein). In the case of an infinite lattice the energy distribution is given by

$$
p(E)=\sum_{i=0}^{z-1} f_{i} \delta\left(E-E_{i}\right),
$$

where $f_{i}$ denotes the statistical weight and $E_{i}$ denotes the position of each peak. It was found numerically that the statistical weights are independent of the input energy $\delta[17$. Thus the statistical weights can be regarded as another fingerprint of the Zhang model.

Analyzing the numerically obtained avalanche distributions [Eq. (4)] it was observed that the avalanche exponents of the Zhang model exhibit finite-size corrections according to [17, 18

$$
\tau_{x}(L)=\tau_{x}-\frac{\text { const }_{\mathrm{x}}}{L} .
$$

In this case the values of the infinite lattice $\tau_{x}$ are obtained by an extrapolation to $L \rightarrow \infty$. More than the explicit values of the exponents this characteristic system size dependence allows us in the following to identify the Zhang like scaling behavior.

\section{THE MANNA MODEL}

A stochastic sandpile model in which integer values represent local energies (or number of sand-grains) was introduced by Manna [6]. Here, unstable sites relax to zero if $E_{\mathbf{r}} \geq E_{\mathrm{c}}$ and the removed energy is randomly distributed to the nearest neighbors in the way that one chooses randomly for each energy unit (one sand-grain) one neighbor. Thus the Manna model is characterized by a stochastic energy transfer and according to [8] it belongs to the so-called universality class of undirected on average sandpile models.

Due to the reduction of the energy of unstable sites to zero both the Zhang and the Manna model are nonAbelian models [19], i.e., the stable energy configurations depend on the sequence in which unstable sites are toppled. Recently Dhar introduced an Abelian version of the two-dimensional Manna model where the energy of critical sites is not reduced to zero but $E_{i, j} \rightarrow E_{i, j}-2$. The energy $\Delta E=2$ is then equally distributed with probability $1 / 2$ to the sites $(i \pm 1, j)$ or otherwise to the sites $(i, j \pm 1)$ 20. In this case it is possible to extend an operator algebra, which was successfully applied in studying the Bak-Tang-Wiesenfeld model [19], to this modified Manna model.

In contrast to this analytically tractable Abelian Manna model we consider in this paper the original Manna model $\left(E_{\underline{r}} \rightarrow 0\right)$. Numerous numerical analysis of the Manna model were performed for $E_{\mathrm{c}}=2$ and the values of the exponents are known within some errorbars (see for instance [6, $8,12,21$ and references therein). Usually one assumes that the value of the critical energy $E_{\mathrm{c}}$ has no influence on the scaling behavior of the model (see for instance [8,21]) and indeed the analysis of the avalanche probability distributions [Eq. (4)] for $E_{\mathrm{c}} \in\{2,3,5,10\}$ reveals that the exponents are independent of $E_{\mathrm{c}}$ (see Fig. 1 in ref. 21]). But as we will see a crossover to a different universality takes place for sufficiently large values of the critical energy.

Consider the Manna model on a lattice with $z$ nearest neighbors. Assume that a given site $\underline{r}$ exceeds the critical value, i.e., $E_{\underline{r}} \geq E_{\mathrm{c}}$. Thus a toppling process takes place and the unstable energy $E_{\underline{r}}$ is randomly distributed to the nearest neighbors. A given neighbor of $\underline{r}$ get $n$ energy units and the corresponding probability distribution is

$$
P\left(n, E_{\underline{r}}\right)=\left(\begin{array}{c}
E_{\underline{r}} \\
n
\end{array}\right) p^{n} q^{E_{\underline{r}}}
$$

with $p=1 / z$ and $q=1-p$. The corresponding expectation value of the energy transfer is $\mu=p E_{\underline{r}}$ and its variance $\sigma=\sqrt{p q E_{\underline{r}}}$. One can therefore distinguish between two regimes: for small values of $E_{\underline{r}}$ the expectation value is of the same order as the variance $(\mu \approx \sigma)$, i.e., the energy transfer to the nearest neighbors is characterized by strong fluctuations. With increasing unstable energies the fluctuations decreases and they can be neglected if $\mu \gg \sigma$ for large values of $E_{\mathrm{r}}$.

Since the value of the unstable energy $E_{\underline{r}}$ is of the same order as the critical energy $E_{\mathrm{c}}$ we introduce the crossover parameter $K=\sqrt{(z-1) / E_{\mathrm{c}}}$. As long as the fluctuations are relevant $K \approx 1$ we expect that the scaling behavior agrees with that of the Manna model for small $E_{\mathrm{c}}$. Increasing the threshold value $E_{\mathrm{c}}$ the fluctuations become irrelevant if $K \ll 1$. In this case the energy is nearly equally distributed to the nearest neighbors and the model corresponds to the Zhang model in the limit $\delta \ll E_{\mathrm{c}}$. Thus we expect that a crossover from the Manna to the Zhang scaling behavior takes place if one increases the value of the critical energy. The details of this crossover are investigated in the next section.

\section{CROSSOVER BEHAVIOR}

\section{A. Energy distribution and energy correlations}

In this subsection we investigate the static properties of the Manna model for various values of the critical energy $E_{\mathrm{c}}$. We show that the crossover affects the distribution of the energies, its scaling behavior as well as the spatial correlations of the energies.

We measured the average energy in the steady state

$$
\langle E\rangle_{L}=\left\langle L^{-2} \sum_{i, j} E_{i, j}\right\rangle
$$

for various values of $L$ and $E_{\mathrm{c}}$. Figure $\mathbb{1}$ shows the system size dependence of the average energy $\langle E\rangle_{L}$ for three 


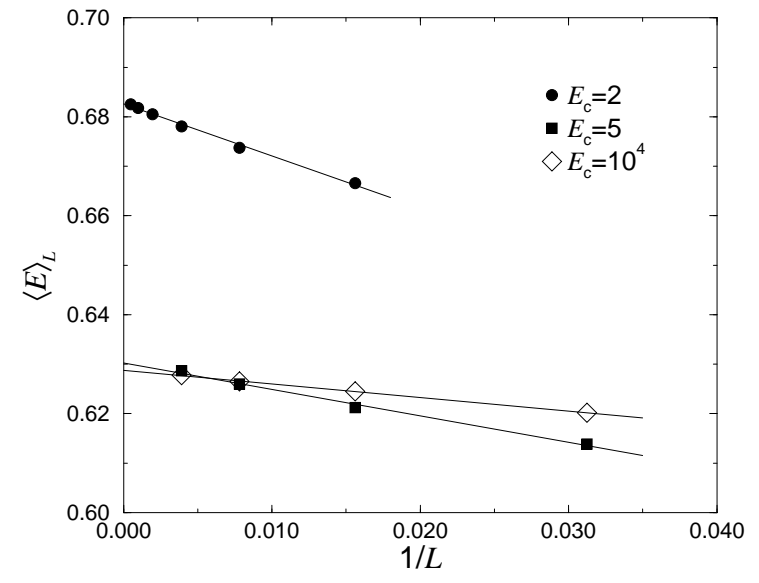

FIG. 1. The average energy in the steady state $\langle E\rangle_{L}$ as a function of the inverse system size $L$ for different values of $E_{\mathrm{c}}$. The extrapolation to the vertical axis yields the value of the average energy for $L \rightarrow \infty$.

different values of $E_{\mathrm{c}}$. For all considered values of $E_{\mathrm{c}}$ we observed that the system size dependence of the average energy is given by

$$
\langle E\rangle_{L}=\langle E\rangle_{L \rightarrow \infty}-\text { const } / L .
$$

This behavior was already found by Manna for $E_{\mathrm{c}}=2$ [6] and it is also known from the Bak-Tang-Wiesenfeld model [22]. The origin of this system size dependence are boundary effects. The average energy on the boundary is smaller than the energy in the bulk and the relative number of boundary sites scales as $L^{-1}$.

The extrapolation to $L \rightarrow \infty$ yields the average energy of the infinite system size and the obtained values are shown in Fig. 2 as a function of $E_{\mathrm{c}}$. Increasing the critical energy from $E_{\mathrm{c}}=2$ the average energy $\langle E\rangle_{L \rightarrow \infty}$ decreases and reaches a minimum for $E_{\mathrm{c}} \approx 100$. Here the

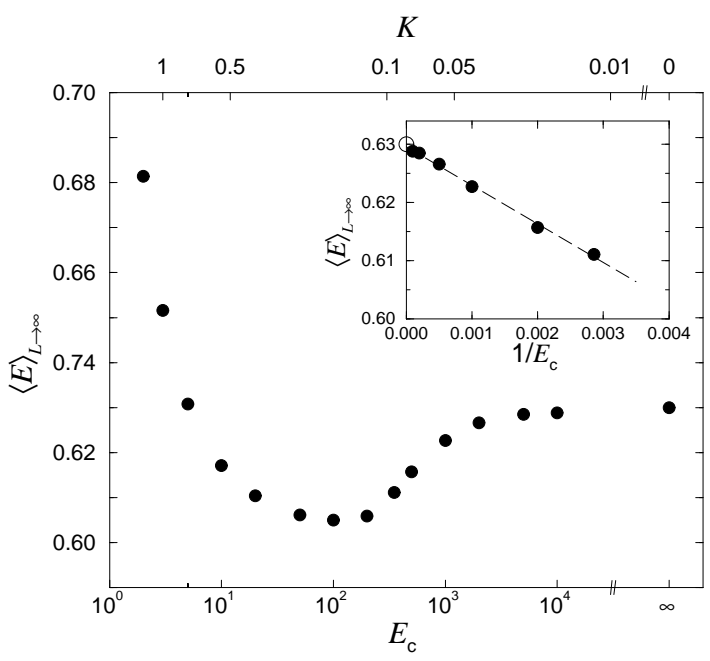

FIG. 2. The average energy in the steady state $\langle E\rangle_{L \rightarrow \infty}$ as a function of the critical energy $E_{\mathrm{c}}$. The inset displays the average energy vs. $1 / E_{\mathrm{c}}$ for $E_{\mathrm{c}} \geq 350$. The corresponding value of the pure Zhang model is marked as $E_{\mathrm{c}}=\infty$ and as an open circle (inset), respectively.
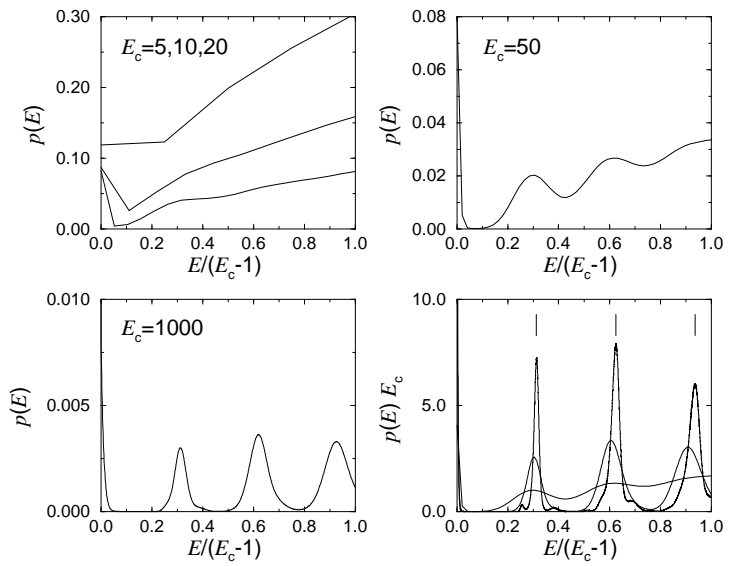

FIG. 3. The energy probability distribution $p(E)$ for $L=128$ and various values of $E_{\mathrm{c}}$. To guide the eye solid lines are plotted instead of symbols. Normalizing $p(E)$ to $E_{\mathrm{c}}$ allows to illustrate how the peak structure of the distribution appears with increasing critical energy. This is shown in the lower right figure for $E_{\mathrm{c}}=50,500,10000$. The vertical solid lines correspond to the position of the peaks of the Zhang model $E_{i}=5 / 16 i$ with $i=0,1,2,3$ [17].

behavior changes and the average energy increases with the critical energy. For large values of $E_{\mathrm{c}}$ the average energy saturates in the vicinity of the value of the Zhang model. A detail analysis suggests that the dependence of average energy on $E_{\mathrm{c}}$ is given by

$$
\langle E\rangle_{L \rightarrow \infty}=\langle E\rangle_{\text {Zhang }}-\text { const } / E_{\mathrm{c}}
$$

for $E_{\mathrm{c}} \geq 350$ (see inset of Fig. 2). Since finite values of the critical energy results in finite fluctuations we get from Eq. (10) that the average energy is affected by these fluctuations even for very large values of $E_{\mathrm{c}}$.

After the average value of the energy we consider now the steady state distribution $p(E)$ of the energies. In
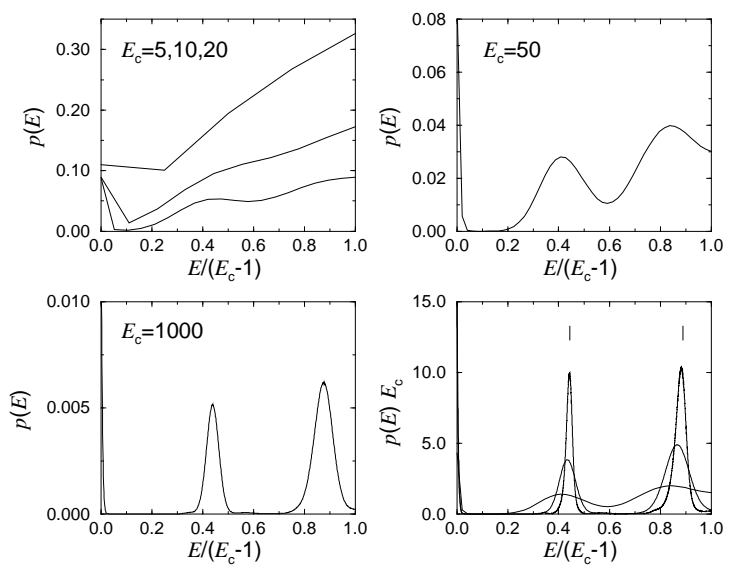

FIG. 4. Analogous to Fig. 3 but for a honeycomb lattice. The vertical solid lines correspond to the position of the peaks of the Zhang model $E_{i}=4 / 9 i$ with $i=0,1,2$ [17. 


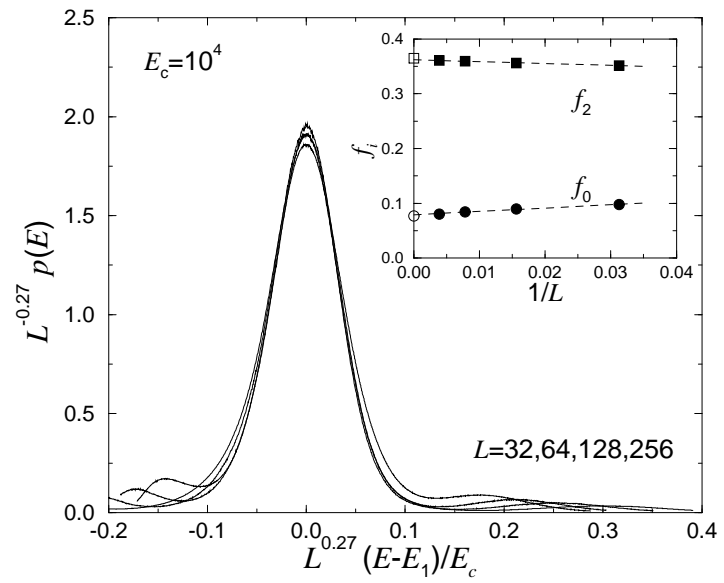

FIG. 5. The finite-size scaling plot of the first maximum of the energy distribution $p(E)$. The inset displays the statistical weights $f_{0}$ and $f_{2}$ as a function of the inverse system size. The values of the infinite system (extrapolation to the vertical axis) agree with those of the Zhang model (open symbols) obtained from 17.

Fig. 3 we plot $p(E)$ for various values of $E_{\mathrm{c}}$. With increasing critical energy a peak structure appears with four distinct peaks located at $E_{i}=5 / 16 i$ with $i=0,1,2,3$. Thus the positions of the peaks agree with the corresponding values of the Zhang model (see [17] and reference therein).

A similar analysis on a honeycomb lattice is shown in Fig. 4 . Here the probability distribution is characterized by three peaks $(z=3)$ located at multiples of $E=4 / 9$. Again these values are in agreement with those of the Zhang model.

It is known from the Zhang model that the maxima of the energy distribution $p(E)$ increase with the system size and that $p(E)$ is characterized by $z \delta$-peaks

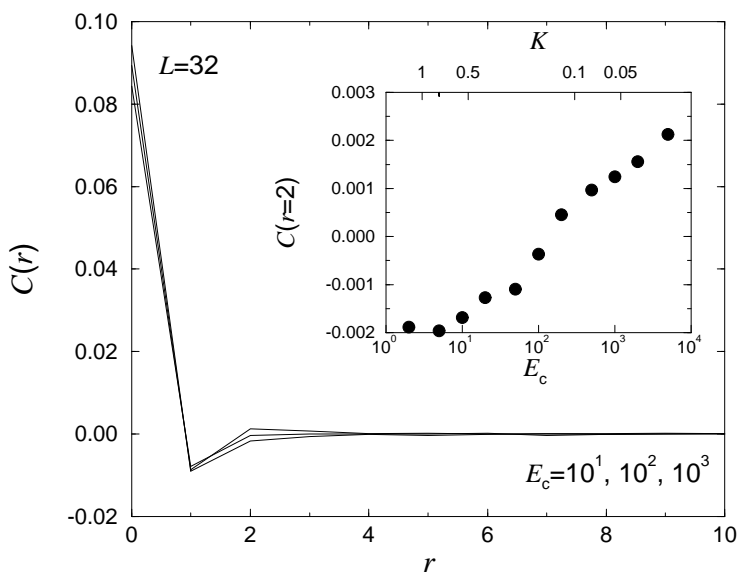

FIG. 6. The correlation function $C(r)$ for different values of the critical energy $E_{\mathrm{c}}$. The inset displays the value $C(r=2)$ which is an indicator for the crossover from the Manna scaling behavior $[C(r=2)<0]$ to the Zhang scaling behavior $[C(r=2)>0]$. for $L \rightarrow \infty$ 17. Therefore we measured the probability distribution $p(E)$ for a large but fixed critical energy $\left(E_{\mathrm{c}}=10^{4}\right)$ and for various system sizes. In Fig. 5 we present the rescaled distribution $L^{y} p(E)$ as a function of $L^{-y}\left(E-E_{i}\right)$ around the first peak at $E_{1}=5 / 16$. The resulting data collapse shows that similar to the pure Zhang model the peak diverges for $L \rightarrow \infty$. But in contrast to the pure Zhang model where the finite-size behavior of $p(E)$ is characterized by an exponent $y \approx 0.6$ [17] we get for $E_{\mathrm{c}}=10^{4}$ the significant different value $y=0.27 \pm 0.1$. Similar results are obtained for the second and third peak (not shown). Further investigations have to show if the finite-size scaling exponent $y$ depends on $E_{\mathrm{c}}$, i.e., if $y$ tends to 0.6 for $E_{\mathrm{c}} \rightarrow \infty$.

But nevertheless the finite-size scaling analysis of the probability distribution yields that $p(E)$ is characterized by four $\delta$-peaks [Eq. (5)] and one can compare the statistical weight $f_{i}$ of each peak with the values of the pure Zhang model. Therefore we divided the interval $\left[0, E_{\mathrm{c}}\right]$ in four parts and measured in each part the area under the curve $p(E)$ for various system sizes. The values of $f_{0}$ and $f_{2}$ are shown in the inset of Fig. 5 as a function of the inverse system size. The same system size dependence was observed for the pure Zhang model [17]. The extrapolation to $L \rightarrow \infty$ yields the statistical weights $f_{i}$ and the obtained values agree with those of the Zhang model (see vertical axis of the inset of Fig. 5).

Finally we consider in this section the energy correlations in the steady state. The correlation function is defined as

$$
C(\underline{r})=\frac{\left\langle E_{\underline{r}^{\prime}} E_{\underline{r}^{\prime}+\underline{r}}\right\rangle-\left\langle E_{\underline{r}^{\prime}}\right\rangle\left\langle E_{\underline{r}^{\prime}+\underline{r}}\right\rangle}{\left(E_{\mathrm{c}}-1\right)^{2}} .
$$

In Fig. 6] we plot $C(r)$ along the symmetry axis of the square lattice. Starting from the autocorrelation peaks at $r=0$ the energies of neighboring sites $(r=1)$ display an anti-correlated behavior. This is caused by the toppling events and is an typical property of sandpile models where the energy of instable sites is reduced to zero. In the inset of Fig. 6 we plot the energy correlations for the distance $r=2$ as a function of $E_{\mathrm{c}}$ and $K$, respectively. For small values of $E_{\mathrm{c}}$ we get negative correlations which increases with the critical energy. In the surrounding of $K^{\star} \approx 0.15$ the behavior changes from an anti-correlated behavior $(C<0)$ to a correlated behavior $(C>0)$. Positive correlations between sites of the same sublattice $(r=2)$ are a typical feature of sandpile models with an isotropic energy transfer to the nearest neighbors. Thus positive correlations within a sublattice indicate a Zhang like behavior whereas negative correlations are a characteristic of the Manna model. The analysis of the avalanche distributions in the next subsection reveals that the model exhibits the typical Manna scaling behavior (for $E_{\mathrm{c}}=2$ ) as long as the energies are anti-correlated, i.e., the scaling behavior changes above the value $K^{\star}$. 


\section{B. Avalanche distributions}

In the following we consider the avalanche distributions [Eq. (4)] and examine the behavior of the corresponding exponents $\tau_{x}$ as a function of the critical energy. Using the reported values of the exponents of the Zhang and Manna (for $E_{\mathrm{c}}=2$ ) model [6, 17, 12, 21] we expect that the difference is nearly $\% 1$ for the size exponent $\tau_{s}$, less than $\% 2$ for $\tau_{a}$, and nearly $\% 5$ for the radius exponent $\tau_{r}$. In the case of the duration exponent it seems that both models are characterized by the value $\tau_{t} \approx 3 / 2$. In the following we analysis the size and radius exponents. The exponents are obtained from a regression analysis of the corresponding probability distribution.

In Fig. 7 we plot the exponent $\tau_{r}$ for different values of the system size $L$ and different critical energies $E_{\mathrm{c}}$ (see also Table II). One can distinguish between three regimes: for small values of the critical energy $\left(E_{\mathrm{c}} \lesssim\right.$ 100) the exponents are independent of $E_{\mathrm{c}}$ and display no significant system size dependence. This is the regime of the Manna universality class and the obtained results agree with the observed universal behavior for $E_{\mathrm{c}} \leq 10$ in 21].

For large values of the critical energy $\left(E_{\mathrm{c}} \gtrsim 5000\right)$ another regime occurs where the exponents are nearly independent of $E_{\mathrm{c}}$ but display a significant system size dependence. These system size dependence agrees with the observed $L$-dependence of the Zhang model [Eq. (6)]. Thus we recover the third fingerprint of the Zhang model for sufficiently large values of $E_{\mathrm{c}}$. We call this regime in the following the Zhang regime. Between the Manna and the Zhang regime we find a third transient regime where the exponents depend strongly on the critical energy and on the system size.

The avalanche size exponent $\tau_{s}$ displays a similar behavior (see Fig. 8 and Table II). Again the Manna regime is characterized by independent exponents. Approach-

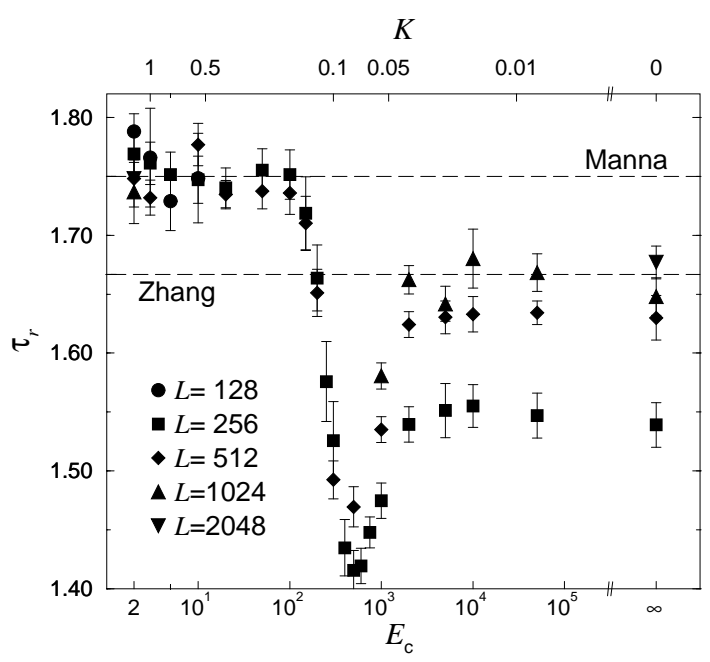

FIG. 7. The avalanche exponent $\tau_{r}$ as a function of the critical energy $E_{\mathrm{c}}$ and the crossover parameter $K$, respectively. The dashed lines indicate the value of the Manna model for $E_{\mathrm{c}}=2$ (upper) and the Zhang (lower) model. The corresponding value of the pure Zhang model is marked as $E_{\mathrm{c}}=\infty$.

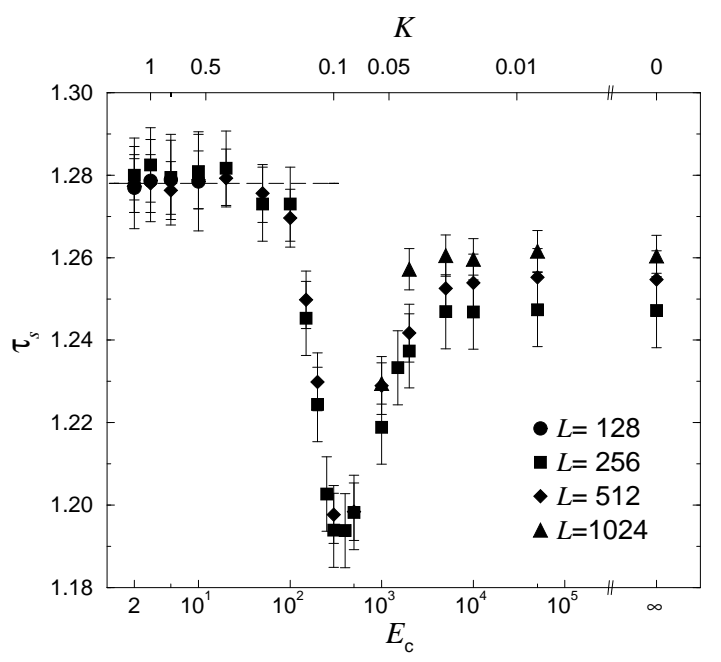

FIG. 8. The avalanche exponent $\tau_{s}$ as a function of the critical energy $E_{\mathrm{c}}$ and the crossover parameter $K$, respectively. The dashed line indicates the value of the Manna model for $E_{\mathrm{c}}=2$. The corresponding values of the pure Zhang model are marked as $E_{\mathrm{c}}=\infty$.

ing a certain value $\left(E_{\mathrm{c}} \approx 100\right)$ the exponent depends strongly on $E_{\mathrm{c}}$ in the transient regime. In the third regime $\left(E_{\mathrm{c}} \gtrsim 5000\right)$ we observe the characteristic Zhang system size dependence. Thus we see that the scaling behavior corresponds to that of the Zhang model for large but finite values of the energy threshold $E_{\mathrm{c}}$. This means that adding stochasticity to the toppling rules of the Zhang model does not force a change of the universality class in all cases. Only a sufficiently large stochasticity is relevant, otherwise it is irrelevant and the scaling behavior is unchanged.

Considering the behavior of the exponents $\tau_{s}$ and $\tau_{r}$ in the transient regime it seems that the exponents increase with the system size. Especially the "over-shooting" ef-



FIG. 9. The avalanche exponent $\tau_{s}$ as a function of the scaling parameter $K$ for the simple cubic (sc) and honeycomb (hc) lattice. The dashed line indicates the value of the Manna model for $E_{\mathrm{c}}=2$ and the arrow marks the value $K^{\star}=0.15$ obtained from the analysis of the correlation function [Eq. (11)]. 
TABLE I. Some values of the avalanche radius exponent $\tau_{r}$ for various values of the critical energy $E_{\mathrm{c}}$ and different sizes $L$ of the square lattice. The corresponding value of the pure Zhang model is marked as $E_{\mathrm{c}}=\infty$.

\begin{tabular}{llll}
\hline \hline$E_{\mathrm{c}}$ & $L=256$ & $L=512$ & $L=1024$ \\
\hline 2 & 1.769 & 1.748 & 1.737 \\
5 & 1.752 & 1.732 & \\
10 & 1.747 & 1.777 & \\
20 & 1.740 & 1.735 & \\
50 & 1.755 & 1.738 & \\
100 & 1.752 & 1.736 & \\
200 & 1.664 & 1.651 & 1.581 \\
500 & 1.416 & 1.470 & 1.662 \\
1000 & 1.475 & 1.535 & 1.642 \\
2000 & 1.539 & 1.624 & 1.680 \\
5000 & 1.551 & 1.630 & 1.648 \\
10000 & 1.555 & 1.633 & \\
50000 & 1.547 & 1.634 & \\
$\infty$ & 1.539 & 1.630 & \\
\hline \hline
\end{tabular}

fect decreases with increasing $L$ and usually one would expect that this regime disappears for $L \rightarrow \infty$. Unfortunately it is impossible to conclude from the numerical results whether the transient regime still exist in the thermodynamic limit. Further investigations are needed to answer this question seriously.

In the last figure we plot the size exponent $\tau_{s}$ for two different lattice types as a function of the crossover parameter $K$. As one can see both curves display the same behavior, i.e., the transition from the Manna regime to the transient regime takes place at $K^{\star} \approx 0.2$ independent of the lattice type. This value is in agreement with the value $K^{\star} \approx 0.15$ obtained from the analysis of the correlation function, i.e., the change of the scaling behavior coincides with the change from negative to positive correlations $C(r=2)$. Thus we conclude that the Manna scaling behavior is strongly connected to the anticorrelations between next nearest neighbors.

TABLE II. Some values of the avalanche size exponent $\tau_{s}$ for various values of the critical energy $E_{\mathrm{c}}$ and different sizes $L$ of the square lattice. The corresponding value of the pure Zhang model is marked as $E_{\mathrm{c}}=\infty$.

\begin{tabular}{llll}
\hline \hline$E_{\mathrm{c}}$ & $L=256$ & $L=512$ & $L=1024$ \\
\hline 2 & 1.280 & 1.278 & 1.279 \\
5 & 1.279 & 1.276 & \\
10 & 1.281 & 1.279 & \\
20 & 1.282 & 1.279 & \\
50 & 1.273 & 1.276 & \\
100 & 1.273 & 1.270 & \\
200 & 1.224 & 1.230 & 1.230 \\
500 & 1.198 & 1.198 & 1.257 \\
1000 & 1.219 & 1.229 & 1.261 \\
2000 & 1.237 & 1.242 & 1.260 \\
5000 & 1.247 & 1.253 & 1.262 \\
10000 & 1.246 & 1.254 & 1.260 \\
50000 & 1.248 & 1.255 & \\
$\infty$ & 1.247 & 1.255 & \\
\hline \hline
\end{tabular}

\section{SUMMARY}

We investigated the scaling behavior of the Manna model as a function of the threshold condition. Increasing the value of the critical energy a crossover takes place from the universality class where the energy transfer is undirected on average to the universality class of an isotropic energy transfer (Zhang model). For sufficiently large thresholds all characteristic of the Zhang model could be recovered, namely the quantization of the energy values in the steady state, the statistical weights of the energy quantums in the thermodynamic limit and the observed system size dependence of the avalanche exponents. Our analysis suggests that the scaling behavior of the stochastic sandpile model is connected to the negative correlations between next nearest lattice sites.

I would like to thank A. Hucht for useful discussions.

* E-mail: sven@thp.uni-duisburg.de

[1] P. Bak, C. Tang, and K. Wiesenfeld, Phys. Rev. Lett. 59, 381 (1987).

[2] B. Drossel and F. Schwabl, Phys. Rev. Lett. 69, 1629 (1992).

[3] B. Drossel, S. Clar, and F. Schwabl, Phys. Rev. E 50, 2399 (1994).

[4] D. Dhar and R. Ramaswamy, Phys. Rev. Lett. 63, 1659 (1989).

[5] B. Tadić and D. Dhar, Phys. Rev. Lett. 79, 1519 (1997).

[6] S. S. Manna, J. Phys. A 24, L363 (1991).

[7] Y.-C. Zhang, Phys. Rev. Lett. 63, 470 (1989).

[8] A. Ben-Hur and O. Biham, Phys. Rev. E 53, R1317 (1996).

[9] K. Christensen and Z. Olami, Phys. Rev. A 46, 1829 (1992).

[10] M. De Menech, A. L. Stella, and C. Tebaldi, Phys. Rev. E 58, 2677 (1998).

[11] C. Tebaldi, M. DeMenech, and A.L. Stella, Phys. Rev. Lett. 83, 3952 (1999).

[12] A. Chessa, H. E. Stanley, A. Vespignani, and S. Zapperi, Phys. Rev. E 59, 12 (1999).

[13] S. Lübeck and K.D. Usadel, Phys. Rev. E 55, 4095 (1997).

[14] L. Pietronero, P. Tartaglia, and Y.-C. Zhang, Physica A 173, 22 (1991).

[15] I. M. Jánosi, Phys. Rev. A 42, 769 (1990).

[16] A. Díaz-Guilera, Phys. Rev. A 45, 8551 (1992).

[17] S. Lübeck, Phys. Rev. E 56, 1590 (1997).

[18] A. Giacometti and A. Díaz-Guilera, Phys. Rev. E 58, 247 (1998).

[19] D. Dhar, Phys. Rev. Lett. 64, 1613 (1990).

[20] D. Dhar, Physica A 270, 69 (1999).

[21] S. Lübeck, Phys. Rev. E 61, 204 (2000).

[22] P. Grassberger and S. S. Manna, J. Phys. (France) 51, 1077 (1990). 